\title{
BMJ Open Epidemiology of multimorbidity among people living with HIV in sub-Saharan Africa: a systematic review protocol
}

\author{
Kelechi Elizabeth Oladimeji (D) , ${ }^{1,2}$ Armstrong Dzomba, ${ }^{3}$ Olatunji Adetokunboh, ${ }^{4,5}$ \\ Lindiwe Zungu, ${ }^{2}$ Sanni Yaya, ${ }^{6}$ Daniel Ter Goon ${ }^{1}$
}

To cite: Oladimeji KE, Dzomba A, Adetokunboh 0, et al. Epidemiology of multimorbidity among people living with HIV in subSaharan Africa: a systematic review protocol. BMJ Open 2020;10:e036988. doi:10.1136/ bmjopen-2020-036988

- Prepublication history for this paper is available online. To view these files, please visit the journal online (http://dx.doi. org/10.1136/bmjopen-2020036988).

Received 14 January 2020 Revised 09 November 2020 Accepted 16 November 2020

Check for updates

(c) Author(s) (or their employer(s)) 2020. Re-use permitted under CC BY-NC. No commercial re-use. See rights and permissions. Published by BMJ.

For numbered affiliations see end of article.

Correspondence to Dr Kelechi Elizabeth Oladimeji; oladimejikelechi@yahoo.com

\section{ABSTRACT}

Introduction Sub-Saharan Africa remains the epicentre of the HIV pandemic, yet enormous knowledge gaps still exist to elicit a comprehensive portrait of multimorbidity and HIV linkage. This study aims to conduct a systematic meta-analysis of peer-reviewed literature to investigate the current status of multimorbidity epidemiology among people living with HIV (PLHIV) in sub-Saharan Africa. Methods and analysis Our review will assess observational studies (ie, cohort, case-control and crosssectional) on multimorbidity associated with HIV/AIDS between 1 January 2005 and 31 October 2020 from sub-Saharan Africa. Databases to be searched include PubMed/MEDLINE, Scopus, Web of Science, Cochrane library, African Index Medicus and African Journals Online. We will also search the WHO clinical trial registry and databases for systematic reviews. The search strategy will involve the use of medical subject headings and key terms to obtain studies on the phenomena of HIV and multimorbidity at high precision. Quality assessment of eligible studies will be ascertained using a validated quality assessment tool for observational studies and risk of bias through sensitivity analysis to identify publication bias. Further, data on characteristics of the study population, multimorbid conditions, epidemiological rates and spatial distribution of multimorbid conditions in PLHIV will be extracted. Heterogeneity of individual studies will be evaluated using the $\mathrm{I}^{2}$ statistic from combined effect size estimates. The statistical analysis will be performed using STATA statistical software V.15 and results will be graphically represented on a forest plot.

Ethics and dissemination Ethical approval is not applicable in this study as it is a systematic review of published literature. The review findings may also be presented at conferences or before other relevant stakeholders.

PROSPERO registration number CRD42020148668.

\section{INTRODUCTION}

Since the discovery of HIV in the 1980s, the global HIV epidemic remains disproportionately concentrated in sub-Saharan Africa such that the region carries the highest HIVrelated morbidity and mortality burden. ${ }^{2-5} \mathrm{In}$ 2018, 68\% of people living with HIV (PLHIV) resided in the region, and an estimated $60 \%$ of deaths were attributable to HIV, ${ }^{6}$ thereby

\section{Strengths and limitations of this study}

Reporting incident, prevalent estimates and trajectories of the multimorbidity burden among people living with HIV across different sociodemographic settings in sub-Saharan Africa epidemic will provide requisite evidence to inform clinical thinking and practice.

- Findings may be integrated into non-communicable diseases surveillance programmes, health systems and delivery for patient-centric prevention and control services of cardiometabolic diseases by national HIV care programmes.

- The novelty of the proposed systematic review study includes being set in a 15-year period (ie, 20052020 ) in a region comprising of low-income and middle-income countries with large HIV burden and knowledge gaps for HIV and multimorbidity.

- Part of the challenge will be defining and ascertaining the presence of multimorbid conditions since individual studies interchangeably use biomarker measurements or self-reports to identify diseases; thus, the interpretive worth of results may be limited by the quality and adequacy of available data.

- Synthesis of literature examining the link between HIV and multimorbidity reflects a necessary focus given the HIV treatment landscape changes in sub-Saharan Africa, a reality often omitted in the development of health research and programmatic responses.

demonstrating the massive scale of the HIV epidemic. As the HIV burden increased over the years, substantial declines in AIDSrelated deaths largely due to survival benefits of combined antiretroviral therapy (ART) was reported..$^{7-9}$ Unfortunately, emerging non-acquired immune-deficiency syndrome (non-AIDS) conditions in HIV-infected patients treated with highly active ART has greatly affected progress made in the reduction of HIV-related deaths. ${ }^{10}$ The implication of these emerging non-AIDS conditions is a devastating increase in the burden of multimorbidity ranging from chronic noncommunicable diseases (NCD) such as 
obesity/overweight and cardiometabolic conditions, chronic cardiovascular diseases, dysfunctional immune systems to concomitant disruptions of cognitive function and mental health conditions. ${ }^{112}$ The huge overlap between killer infections such as HIV, tuberculosis (TB), with NCDs like hypertension and diabetes mellitus (DM) results in a 'double burden of disease' in loe-income and middle-income countries. ${ }^{13}$ This double burden of disease as explained by Bates $e a^{13}{ }^{13}$ can also be referred to as multimorbidity (as defined below), and this causes a huge health problem in PLHIV in sub-Saharan Africa.

The term multimorbidity is defined as the coexistence of two or more chronic conditions, where each must be an NCD, a mental health disorder or an infectious disease of long duration. ${ }^{14} 15$ Global health data show that Africa, compared with other continents, suffers more from the combined presence and effects of NCDs together with HIV/AIDS and $\mathrm{TB}^{16}$ The interactions between these multimorbid conditions and their treatment pose many challenges to the effective management of PLHIV; a major challenge in managing PLHIV and multimorbidity is attributed to polypharmacy. One review, for example, noted that polypharmacy in older adults could lead to toxic drug interactions and/or ineffectiveness in achieving therapeutic effects, as well as falls, increased resource utilisation and higher mortality. ${ }^{17}$ The prevalence of adverse interactions of ART and non-HIV medications has been linked to potentially serious drug toxicities and interruptions in ART. ${ }^{18} 19$ Further, optimal management of patients with multimorbidity is known to be complicated by economic, social, emotional and psychological issues, coupled with declining functional capacity and increasing need for multiple medications and health specialty providers for patients. ${ }^{20}{ }^{21}$ Consequences of these challenges to the optimal management of multimorbidity include poorer clinical outcomes, increased hospitalisation rates and concomitant healthcare expenditure and frequency of service use featuring high long-term dependence on secondary than primary care. $^{22-25}$

Currently, there is paucity of evidence using literaturebased synthesis to evaluate the occurrence of multimorbidity in PLHIV from sub-Saharan Africa. The studies providing evidence on HIV morbidities from settings in sub-Saharan Africa, ${ }^{26-28}$ are majorly observational studies and fewer compared with the larger literature from high-income countries, ${ }^{11}{ }^{29-35}$ however, most sampling an ageing population. Given the detrimental effects of emerging multimorbidity within the context of longterm survival with HIV, there is a need to synthesise available evidence to determine the trends and magnitude of multimorbidity among PLHIV. An effort of this scale will help identify existing gaps and provide strategies for strengthening health systems for effective management of comorbid conditions in PLHIV from sub-Saharan Africa and other geographical areas where HIV is prevalent. Thus, the proposed systematic review aims to examine the epidemiology of multimorbidity in the HIV-infected population in sub-Saharan Africa. To this end, the questions that this review seeks to answer, and specific objectives are outlined below.

\section{Review questions}

1. What are the multimorbid conditions affecting PLHIV in sub-Saharan Africa?

2. What is the incidence and prevalence of multimorbidity in PLHIV in sub-Saharan Africa?

3. What is the spatial distribution of HIV multimorbidity across sub-Saharan Africa?

\section{Specific objectives}

1. To profile the multimorbid diseases among PLHIV in sub-Saharan Africa.

2. To determine the incidence and prevalence of multimorbidity among PLHIV in Sub Saharan Africa.

3. To assess the spatial distribution of HIV associated multimorbidity across sub-Saharan Africa.

\section{METHODS AND ANALYSIS}

\section{Study design}

The systematic review and meta-analysis protocol will follow the Joanna Briggs Institute online manual ${ }^{36}$ and Meta-analysis of observational studies in epidemiology (MOOSE) guidelines. ${ }^{37}$

\section{Eligibility criteria}

In order to systematically identify the relevant primary studies for this review and minimise the potential for bias, we will define the outcome of interest and develop an inclusion/exclusion criterion as discussed below. There are different frameworks used to define and understand the phenomena of multimorbidity globally, ${ }^{38}{ }^{39}$ however, for our working definition, we will adopt the definition mentioned above for multimorbidity by WHO and The Lancet. ${ }^{14} 15$ Accordingly, the inclusion and exclusion criteria for studies in the systematic review will be guided by the following factors:

\section{Inclusion criteria}

The studies will be selected for inclusion using the PICOTS framework explained by Lackey, ${ }^{40}$ where; $\mathrm{P}$ is Population, I imply Intervention, $\mathrm{C}$ is Comparison, $\mathrm{O}$ is Outcome, $\mathrm{T}$ is Time frame and $\mathrm{S}$ is Study setting. However, in this review, we will adopt elements of the framework to suit our study design by replacing intervention (I) with exposures (E), that is, PECOTS.

\section{Population}

Studies whose population include PLHIV prior to diagnosis or development of the multimorbid conditions will be included for review. In addition, PLHIV that have commenced ART prior to diagnosis or development of the multimorbid conditions will also be included.

\section{Exposures of interest}

The exposures of interest in this study will be HIV infection and ART. The ascertainment of exposure to HIV and 
ART including whether HIV tests were performed, or initiation of ART was known from patient records versus self-reported including definitions of multiple disease diagnoses will be based on information provided in the eligible studies. Importantly, it has been shown that, despite the survival benefits of ART, treatment confers excess risk for multimorbid conditions among PLHIV. ${ }^{27} 3541$ Hence, the study population will include PLHIV prior to diagnosis or development of the multimorbid conditions. In addition, PLHIV that have commenced ART prior to diagnosis or development of the multimorbid conditions will also be included. Exposure to ART will be defined as HIV positive individuals initiated on ART and linked to HIV care.

\section{Comparison}

The comparator group will include HIV negative individuals if available in studies to be included for review.

\section{Outcomes}

The outcome of this review is the development of multimorbidity due to HIV and also ART, where data are available. The selection of articles for synthesis will be based on whether reports on co-occurring morbid conditions available following HIV infection in PLHIV are and PLHIV that started ART, respectively. Multimorbid conditions such as $\mathrm{TB}$, hypertension, obesity, DM, asthma and depression will be considered, with definition of diseases derived from self-reported diagnosis and use of chronic disease medication, medical records and clinical results in the case of population-specific health screening. The International Classification of Diseases version 9 system for coding and classifying morbidity data from inpatient and outpatient records, physician offices and population-specific surveys will provide an overall basis for disease diagnosis.

Mortality, the secondary outcome will be verified from aggregated cause of death data from individual studies (ie, verbal autopsies) and hospital-level reports subject to the availability of information.

\section{Time frame}

Studies on multimorbidity in the context of HIV published between 1 January 2005 and 31 October 2020 from sub-Saharan Africa will be included. The year 2005 was chosen as the starting point for this review period because that is when most African national HIV programmes had access to subsidised ART through international donors and the US President's Emergency Plan For AIDS Relief which was created in 2003 as a US\$15 billion 5-year plan to combat AIDS. ${ }^{42} 43$

\section{Study setting}

The study setting for this review is the African continent, particularly sub-Saharan Africa.

In addition to our inclusion criteria explained above, study designs of eligible studies will be limited to that of peer-reviewed epidemiological studies (cross-sectional, case-control and cohort studies). Further, only studies published in English language will be considered.

\section{Exclusion criteria}

Non-empirical (such as conceptual papers and opinion pieces), qualitative studies and epidemiological studies which do not report rates and measures of association such as prevalence, incidence ORs and/or adjusted ORs, HRs/or adjusted HRs quantifying the increase in the risk of additional health conditions due to HIV infection will be excluded.

\section{Information sources and search strategy}

We will perform a comprehensive search for eligible studies from databases such as PubMed/MEDLINE, Scopus, African Index Medicus, African Journals Online, Web of Science and Cochrane library. We will also search the WHO clinical trial registry and databases for systematic reviews. The search strategy will involve the use of medical subject headings to elicit studies at high precision, and key terms typically used to express the phenomena of HIV and multimorbidity (online supplemental appendix 1). Overall, only studies meeting the eligibility criteria outlined above will be included in the review.

\section{Study selection}

The title and abstracts of studies on HIV and multimorbidity will be screened for eligibility and data extraction by two of this review authors, (KEO and $\mathrm{AD}$ ). The two reviewers will independently screen the title and abstract of retrieved articles after duplicates has been removed using Microsoft excel and Endnote V.9. Next, the full text of articles selected after screening titles and abstract will be retrieved for further data extraction. During the study selection process, if any disagreements arise (as often is the case) a third author (OA) will be consulted for expert advice and a consensus. In the event of unclear or missing data, we would contact the corresponding authors of these studies through email. If there is no reply from the corresponding authors of these articles after a period of 2 weeks, we will phone (if a phone number can be reached) and also send a reminder email. If there is still no response, the articles requiring further details from the corresponding authors will be excluded and reported in the Preferred Reporting Items for Systematic Reviews and Meta-Analyses flow diagram.

\section{Data extraction}

Data extraction from eligible selected studies will be conducted independently by two reviewers (KEO and $\mathrm{AD})$. On completion of the data extraction process by both $\mathrm{KEO}$ and $\mathrm{AD}$, the data extracted will be discussed at a meeting with the third reviewer (OA). This third reviewer $(\mathrm{OA})$ will examine the extracted data to identify errors or inconsistencies that need to be corrected if necessary. Data to be extracted will include; author's details, year of publication, type of study, geographical setting, HIV status of the study population, study population age, gender, sample size, ART treatment status, incidence and prevalence of multimorbidity, mortality and potential confounders (online supplemental appendix 
2). To ensure the quality and validity of included studies for review, we discuss how methodological rigour will be achieved below.

\section{Quality assessment}

As part of ongoing quality assessment, two reviewers (KEO and $\mathrm{AD}$ ) will independently review and validate eligible articles for further synthesis. In the event of the two reviewers disagreeing on the final critical appraisal, a third reviewer would be consulted. For the quality assessment process, we will use the Newcastle-Ottawa Scale (NOS) for assessing the quality of non-randomised studies in meta-analyses (online supplemental appendix 3). The scoring system has a 'star' structure for assessing the quality of non-randomized studies in three broad areas: the selection of study groups; comparability of groups; and determination of either exposure or outcome of interest. ${ }^{44}$ Following the coding process in the quality assessment report by Roa $e t a l^{45}$ and the decision rule for application of the NOS, ${ }^{46}$ we will assign a star to each of the eight questions (with yes $=1$ star and no $=0$ stars). In principle, studies scoring 5-8 stars will be rated as high quality and those between 1-4 stars, low quality.

\section{Risk of bias}

Further ascertainment of risk of bias will include conducting sensitivity analysis, determining the status of each individual study by how they graphically fit on a funnel plot. Ideally in the funnel plot, studies with high precision plot closer to the average while those with low precision spread evenly on either side of the average, producing a near funnel-shaped distribution. As such, variation from this shape will suggest publication bias. The checklist for MOOSE in online supplemental appendix 4 will be applied to provide details of how background and search strategies including methods, results, discussions and conclusions would be reported.

\section{Outcome(s) measures and data synthesis}

The outcomes for this review will include; types of multimorbid conditions, prevalence and incidence rate of multimorbid conditions and spatial distribution of multimorbid conditions among PLHIV in sub-Saharan Africa. Qualitatively, data will be thematically synthesised; this will involve the systematic coding of data and the development of descriptive and analytical themes. This is a particularly robust approach given our aim to determine HIV coinfections contained in individual studies. First, data extracted through the rubric in online supplemental appendix 2 will be classified according to its content.

Quantitatively, we will conduct meta-analysis using a random-effects model because we expect heterogeneity in the eligible studies to be synthesised. Heterogeneity of individual studies will be evaluated using the $\mathrm{I}^{2}$ statistic from combined effect size estimates in our meta-analysis. A forest plot will be used to graphically present the extent of heterogeneity of synthesised studies in this review. For the random-effects model, we will use the restricted maximum likelihood method to estimate the parameters. As such, we will solely restrict our data extraction to dichotomous data amenable to meta-analysis including prevalence point estimates, ORs and HRs, incidence rate ratios and their corresponding 95\% CIs from profiled multimorbidity. Outcome measures will be stratified by HIV and ART status if data are available. Further, we will estimate the overall incidence and prevalence of each multimorbid conditions (see online supplemental appendices 5 and 6 ) among those who were HIV positive compared with those that were HIV negative based on a random-effects model with combined effects estimates being calculated through meta-analyses. Prior to estimating pooled-effects of the incidence and prevalence data, the Freeman-Tukey double arcsine transformation would be performed to keep at a minimum the variance in incidence and prevalence data from synthesised studies. Funnel plot and Egger's test will be carried out to ascertain publication bias. All statistical analysis will be performed with STATA V.15 statistical software.

\section{Analysis of subgroups or subsets}

Subgroup analysis on articles retrieved from 1 January 2005 to 31 October 2020 will be performed by countries in sub-Saharan Africa, countries in the WHO Africa region, and HIV multimorbid conditions based on the availability of data and quality of included studies. This subgroup analysis will explore demographic factors (age groups and gender), ART treatment and study period.

\section{Patient and public involvement}

Patients or the public were not involved in the design, or conduct, or reporting, or dissemination plans of our research.

\section{DISCUSSION}

In tandem with major social transformations such as urbanization across settings in sub-Saharan Africa, health transition has accelerated in recent years against the background of an evolving HIV treatment and prevention landscape thereby worsening the disease burden of HIV patients. ${ }^{86478}$ The resultant effects are the widespread emergence of an NCDs epidemic as a result of the overall ageing of the HIV infected population and mental disorders especially among the young and middle-aged populations given the early age of infection. ${ }^{49}$ Consequently, our intent for this study is to summarize the totality of evidence linking HIV and multimorbidity risk in sub-Saharan Africa. The study aim is consistent with the Academy of Medical Sciences UK current funding model and approach to address the global challenge of multimorbidity which involves measuring the prevalence of multimorbidity, identifying key diseases including understanding severity of regional and national multimorbidity epidemics as an important initial first step towards developing evidence-based strategies adaptable to healthcare systems. ${ }^{50}$ Findings may in one part be useful in identifying 
further research priorities to address gaps these gaps and on the other support better understanding of the major dimensions predicting multimorbidity such as biological factors (age, gender), social factors and health systems factors (polypharmacy due to treatment of HIV and occurring morbidities) by providing the adequate disaggregated data.

\section{Ethics and dissemination}

Ethical approvals will not be required because this is a review. After completion of the review, findings will be disseminated across the scientific community through publishing in peer-reviewed journals; presentations at conferences, seminars and meetings.

\section{Author affiliations}

${ }^{1}$ Faculty of Health Sciences, University of Fort Hare, East London, South Africa ${ }^{2}$ College of Graduate Studies, University of South Africa, Johannesburg, South Africa ${ }^{3}$ Medical Research Council/Wits Rural Public Health and Health Transitions Research Unit (Agincourt), School of Public Health, Faculty of Health Sciences, University of the Witwatersrand, Johannesburg, South Africa

${ }^{4}$ DST-NRF Centre of Excellence in Epidemiological Modelling and Analysis (SACEMA), Stellenbosch University, Stellenbosch, South Africa

${ }^{5}$ Division of Epidemiology and Biostatistics, Faculty of Medicine and Health Sciences, Stellenbosch University, Cape Town, Western Cape, South Africa

${ }^{6}$ Faculty of Social Sciences, University of Ottawa, Ottawa, Ontario, Canada

Contributors KEO conceived the study; $A D, K E O$ and $O A$ developed the search strategy; KEO and AD drafted the protocol. KEO, AD, DTG, LZ, OA and SY constructively reviewed the manuscript to improve the methodological and intellectual content. All authors read and approved the final manuscript.

Funding The authors have not declared a specific grant for this research from any funding agency in the public, commercial or not-for-profit sectors.

Competing interests None declared.

Patient consent for publication Not required.

Provenance and peer review Not commissioned; externally peer reviewed.

Supplemental material This content has been supplied by the author(s). It has not been vetted by BMJ Publishing Group Limited (BMJ) and may not have been peer-reviewed. Any opinions or recommendations discussed are solely those of the author(s) and are not endorsed by BMJ. BMJ disclaims all liability and responsibility arising from any reliance placed on the content. Where the content includes any translated material, BMJ does not warrant the accuracy and reliability of the translations (including but not limited to local regulations, clinical guidelines, terminology, drug names and drug dosages), and is not responsible for any error and/or omissions arising from translation and adaptation or otherwise.

Open access This is an open access article distributed in accordance with the Creative Commons Attribution Non Commercial (CC BY-NC 4.0) license, which permits others to distribute, remix, adapt, build upon this work non-commercially, and license their derivative works on different terms, provided the original work is properly cited, appropriate credit is given, any changes made indicated, and the use is non-commercial. See: http://creativecommons.org/licenses/by-nc/4.0/.

ORCID iD

Kelechi Elizabeth Oladimeji http://orcid.org/0000-0002-0246-5595

\section{REFERENCES}

1 Why the epidemic is not over. Available: https://www.who.int/hivaids/latest-news-and-events/why-the-hiv-epidemic-is-not-over

2 Dwyer-Lindgren L, Cork MA, Sligar A, et al. Mapping HIV prevalence in sub-Saharan Africa between 2000 and 2017. Nature 2019;570:189-93.

3 Dillon DG, Gurdasani D, Riha J, et al. Association of HIV and art with cardiometabolic traits in sub-Saharan Africa: a systematic review and meta-analysis. Int J Epidemiol 2013;42:1754-71.
4 James SL, Abate D, Abate KH, et al. Global, regional, and national incidence, prevalence, and years lived with disability for 354 diseases and injuries for 195 countries and territories, 1990-2017: a systematic analysis for the global burden of disease study 2017 . The Lancet 2018;392:1789-858.

5 Roth GA, Abate D, Abate KH, et al. Global, regional, and national age-sex-specific mortality for 282 causes of death in 195 countries and territories, 1980-2017: a systematic analysis for the global burden of disease study 2017. The Lancet 2018;392:1736-88.

6 InJoint United Nations Programme on HIV/AIDS (UNAIDS). Unaids data 2019: state of the epidemic. Geneva: UNAIDS, 2019.

7 Bor J, Herbst AJ, Newell M-L, et al. Increases in adult life expectancy in rural South Africa: valuing the scale-up of HIV treatment. Science 2013;339:961-5.

8 Hontelez JAC, Lurie MN, Newell M-L, et al. Ageing with HIV in South Africa. AIDS 2011;25:1665-7.

9 Kharsany ABM, Karim QA. Hiv infection and AIDS in sub-Saharan Africa: current status, challenges and opportunities. Open AIDS J 2016;10:34-48

10 Perdomo-Celis F, Taborda NA, Rugeles MT. CD8 ${ }^{\mathrm{Cd} 8+}$ T-cell response to HIV infection in the era of antiretroviral therapy. Front Immunol 2019;10:10.

11 Archer T. Hiv co-morbidities and Multi-Morbidities. HIV Curr Res 2016;01:2.

12 Chang AY, Gómez-Olivé FX, Payne C, et al. Chronic multimorbidity among older adults in rural South Africa. BMJ Glob Health 2019; 4:e001386.

13 Bates M, Marais BJ, Zumla A. Tuberculosis comorbidity with communicable and noncommunicable diseases. Cold Spring Harb Perspect Med 2015;5:a017889.

14 The Lancet. Making more of multimorbidity: an emerging priority. The Lancet 2018;391:1637.

15 World Health Organization. Multimorbidity: technical series on safer primary care. Geneva: World Health Organization, 2016.

16 Peer $\mathrm{N}$. The converging burdens of infectious and noncommunicable diseases in rural-to-urban migrant sub-Saharan African populations: a focus on HIV/AIDS, tuberculosis and cardiometabolic diseases. Trop Dis Travel Med Vaccines 2015;1:6.

17 Gleason LJ, Luque AE, Shah K. Polypharmacy in the HIV-infected older adult population. Clin Interv Aging 2013;8:749-63.

18 Holtzman C, Armon C, Tedaldi E, et al. Polypharmacy and risk of antiretroviral drug interactions among the aging HIV-infected population. J Gen Intern Med 2013;28:1302-10.

19 Krentz HB, Gill MJ. The impact of Non-Antiretroviral polypharmacy on the continuity of antiretroviral therapy (art) among HIV patients. AIDS Patient Care STDS 2016;30:11-17.

20 Koch G, Wakefield BJ, Wakefield DS. Barriers and facilitators to managing multiple chronic conditions: a systematic literature review. West J Nurs Res 2015;37:498-516.

21 Halman M, Carusone SC, Stranks S, et al. Complex care needs of patients with late-stage HIV disease: a retrospective study. AIDS Care 2014;26:320-5.

22 Bähler C, Huber CA, Brüngger B, et al. Multimorbidity, health care utilization and costs in an elderly community-dwelling population: a claims data based observational study. BMC Health Serv Res 2015;15:23.

23 Lee JT, Hamid F, Pati S, et al. Impact of noncommunicable disease multimorbidity on healthcare utilisation and out-of-pocket expenditures in middle-income countries: cross sectional analysis. PLoS One 2015;10:e0127199.

24 Mavaddat N, Valderas JM, van der Linde R, et al. Association of self-rated health with multimorbidity, chronic disease and psychosocial factors in a large middle-aged and older cohort from general practice: a cross-sectional study. BMC Fam Pract 2014;15:185.

25 Pati S, Swain S, Hussain MA, et al. Prevalence and outcomes of multimorbidity in South Asia: a systematic review. BMJ Open 2015;5:e007235.

26 Oni T, Youngblood E, Boulle A, et al. Patterns of HIV, TB, and noncommunicable disease multi-morbidity in peri-urban South Africa- a cross sectional study. BMC Infect Dis 2015;15:20.

27 Magodoro IM, Esterhuizen TM, Chivese T. A cross-sectional, facility based study of comorbid non-communicable diseases among adults living with HIV infection in Zimbabwe. BMC Res Notes 2016;9:379.

28 Weimann A, Dai D, Oni T. A cross-sectional and spatial analysis of the prevalence of multimorbidity and its association with socioeconomic disadvantage in South Africa: a comparison between 2008 and 2012. Soc Sci Med 2016;163:144-56.

29 De Francesco D, Underwood J, Bagkeris E, et al. Risk factors and impact of patterns of co-occurring comorbidities in people living with HIV. AIDS 2019;33:1871-80. 
30 De Francesco D, Verboeket SO, Underwood J, et al. Patterns of co-occurring comorbidities in people living with HIV. In: Open forum infectious diseases: 2018. US: Oxford University Press, 2018: 5. ofy272.

31 Gallant J, Hsue PY, Shreay S, et al. Comorbidities among US patients with prevalent HIV Infection-A trend analysis. $J$ Infect Dis 2017;216:1525-33.

32 Guaraldi G, Malagoli A, Calcagno A, et al. The increasing burden and complexity of multi-morbidity and polypharmacy in geriatric HIV patients: a cross sectional study of people aged $65-74$ years and more than 75 years. BMC Geriatr 2018;18:99.

33 Maciel RA, Klück HM, Durand M, et al. Comorbidity is more common and occurs earlier in persons living with HIV than in HIV-uninfected matched controls, aged 50 years and older: a cross-sectional study. Int J Infect Dis 2018:70:30-5.

34 Rodriguez-Penney AT, ludicello JE, Riggs PK, et al. Co-Morbidities in persons infected with HIV: increased burden with older age and negative effects on health-related quality of life. AIDS Patient Care STDS 2013;27:5-16.

35 Wong C, Gange SJ, Moore RD, et al. Multimorbidity among persons living with human immunodeficiency virus in the United States. Clinical Infectious Diseases 2018;66:1230-8.

36 Aromataris E, Munn Z. Joanna Briggs Institute reviewer's manual. The Joanna Briggs 2017;299.

37 Stroup DF, Berlin JA, Morton SC, et al. Meta-Analysis of observational studies in epidemiology: a proposal for reporting. Jama 2000;283:2008-12.

38 Hofman K. Prevalence, burden, determinants and risk factors addressing the global challenges of multimorbidity - lessons from South Africa conference proceedings 2016.

39 Mercer S, Furler J, Moffat K, et al. Multimorbidity: technical series on safer primary care: World Health organization 2016.
40 Lackey M. Systematic reviews: Searching the literature [PowerPoint slides], 2013. Available: http://guides.lib.unc.edu/ld.php?content_id= 258919]

41 Deeks SG, Lewin SR, Havlir DV. The end of AIDS: HIV infection as a chronic disease. The Lancet 2013;382:1525-33.

42 A timeline of HIV/AIDS. Available: https://www.hiv.gov/sites/default/ files/aidsgov-timeline.pdf

43 Taylor G. Rolling out HIV antiretroviral therapy in sub-Saharan Africa: 2003-2017. Can Commun Dis Rep 2018;44:68-70.

44 Wells GA, Shea B, O'Connell D, et al. The Newcastle-Ottawa Scale (NOS) for assessing the quality of nonrandomised studies in metaanalyses. Ottawa Hospital Research Institute, 2019. Available: http:// wwwohrica/programs/clinical_epidemiology/oxfordasp

45 Rao SG, Galaviz KI, Gay HC, et al. Factors associated with excess myocardial infarction risk in HIV-infected adults: a systematic review and meta-analysis. J Acquir Immune Defic Syndr 2019;81:224-30.

46 Hartling L, Hamm M, A: M. Validity and inter-rater reliability testing of quality assessment instruments appendix $\mathrm{E}$, decision rules for application of the Newcastle-Ottawa scale 20122017.

47 Gaziano TA, Abrahams-Gessel S, Gomez-Olive FX, et al. Cardiometabolic risk in a population of older adults with multiple co-morbidities in rural South Africa: the HAALSI (health and aging in Africa: longitudinal studies of indepth communities) study. BMC Public Health 2017;17:206.

48 Jardim TV, Reiger S, Abrahams-Gessel S, et al. Hypertension management in a population of older adults in rural South Africa. $J$ Hypertens 2017;35:1283-9.

49 Lalkhen H, Mash R. Multimorbidity in non-communicable diseases in South African primary healthcare. S Afr Med J 2015;105:134.

50 Academy of science of South Africa: addressing the global challenges of multimorbidity-lessons from South Africa 2016. 